\title{
Reduction to the First Order Systems of the Kowalevskian Systems in the Sense of Volevic
}

By

\author{
Masatake MIYAKE*
}

\section{$\S$ I. Intriroduction}

Let the system $L\left(x, t ; \partial_{x}, \partial_{t}\right)$ be given by

$$
L\left(x, t ; \partial_{x}, \partial_{t}\right)=\partial_{t} I_{N}-P\left(x, t ; \partial_{x}\right),
$$

where $P\left(x, t ; \partial_{x}\right)=\left(p_{i j}\left(x, t ; \partial_{x}\right)\right)_{i, j=1, \cdots, N},(x, t)=\left(x_{1}, \cdots, x_{n}, t\right) \in \mathbb{R}^{n+1}$ (or $\left.\mathbb{C}^{n+1}\right), \partial_{x}=\left(\frac{\partial}{\partial x_{1}}, \cdots, \frac{\partial}{\partial x_{n}}\right)$ and $\partial_{t}=\frac{\partial}{\partial t}$.

First, let us consider the case where $(x, t) \in \mathbb{C}^{n+1}$. In this case our considerations are restricted in the holomorphic functions defined in a neighbourhood of the origin of $\mathbb{C}^{n+1}$, so we assume that the coefficients of $p_{i j}$ are holomorphic in $\mathscr{Q}=\sum_{k=1}^{n}\left\{\left|x_{k}\right|<r_{k}\right\} \times\left\{|t|<r_{0}\right\}$. Now we say the system $L$ given by (1.1) is Kowalevskian in $\Omega$ if the theorem of CauchyKowalevski holds at every point in $\Omega$ for $L$. Then in the previous paper [1] the author obtained a necessary and sufficient condition for $L$ to be kowalevskian in $\Omega$ in the case of $n=1$. That is,

Theorem 耳. (Theorem 2 in [1]) ${ }^{1}$. Let $n=1$. Then the system $L$ given by (1.1) is Kowalevskian in $\Omega$ if and only if there exists an operator $J\left(x, t ; \partial_{x}\right)$ of $N \times N$ matrix with following properties: (i) The coefficients of $J$ are meromorphic in $\Omega$. (ii) $J\left(x, t ; \partial_{x}\right)$ is invertible, that is, there exists $J^{-1}\left(x, t ; \partial_{x}\right)$ such that $J^{-1} J=J J^{-1}=I_{N}$. (iii) The systein $J^{-1} L J$ is Kowalevskian in Volevič's sense.

Our purpose in this note is to reduce the kowalevskian system in

Communicated by S. Matsuura, February 7, 1977.

* Department of Mathematics, College of General Education, Nagoya University.

1) For more detailed results, see [1]. 
Volevič's sense to the equivalent first order system by equivalent transformations of systems defined later. Here the equivalent transformation means that under such transformation the well posedness of the Cauchy problem is preserved for example in the class of $C^{\infty}$.

Usually the existence theorem of Cauchy-Kowalevski is proved by the method of approximation (or the method of majorant), and the Kowalevskian system in Volevič's sense is the best possible class in order to prove the convergence of the approximate solutions (see Gåding [2]). Theorem 1 asserts that the Kowalevskian system in Volevičs sense is very reasonable class considering the Cauchy problem in a sense.

Perhaps it was believed that the Kowalevskian system in Volevič's sense is wider class than the first order system. So in this note we shall prove that the two classes in the above are equivalent under a suitable transformation.

Now remember the definition of the Kowalevskian system in Volevič's sense. We say the system $L$ given (1.1) is Kowalevskian in Volevič's sense if

(1. 2) $\operatorname{order} p_{i j}\left(x, t ; \partial_{x}\right) \leqq t_{i}-t_{j}+1$ for any $i, j=1,2, \cdots, N$, where $\left\{t_{i}\right\}_{i=1}^{N} \subset \mathbb{Z}$ and $\mathbb{Z}$ denotes the set of integers.

Next we shall give the definitions of equivalent transformations of systems.

(I) Let the system $L\left(x, t ; \partial_{x}, \partial_{t}\right)$ be the one given by (1.1) and let $\widetilde{L}\left(x, t ; \partial_{x}, \partial_{t}\right)$ be the system given by

$$
\widetilde{L}\left(x, t ; \partial_{x}, \partial_{t}\right)=\partial_{t} I_{N+1}-Q\left(x, t ; \partial_{x}\right),
$$

where $Q$ is the operator of $(N+1) \times(N+1)$ matrix given by

$$
Q\left(x, t ; \partial_{x}\right)=\left(\begin{array}{cc}
P\left(x, t ; \partial_{x}\right) & \vec{c}\left(x, t ; \partial_{x}\right) \\
\overrightarrow{0} & 0
\end{array}\right),
$$

where $\vec{c}\left(x, t ; \partial_{x}\right)={ }^{t}\left(c_{1}\left(x, t ; \partial_{x}\right), \cdots, c_{N}\left(x, t ; \partial_{x}\right)\right), \quad \overrightarrow{0}=(\overbrace{0, \cdots, 0}^{N}$, and $c_{i}\left(x, t ; \partial_{x}\right)$ are differential operator of any order with smooth coefficients, where smooth means $C^{\infty}$ or holomorphic according to the context. Then it is obvious that $L$ is Kowalevskian (or well posed for the Cauchy problem) if and only if $\widetilde{L}$ is Kowalevskian (or well posed for the Cauchy 
problem).

(II) Let $J\left(\partial_{x}\right)$ be the differential operator of $N \times N$ matrix with constants coefficients. We assume that $J\left(\partial_{x}\right)$ is invertible, that is, there exists an inverse operator $J^{-1}\left(\partial_{x}\right)$. Let $\widetilde{L}\left(x, t ; \partial_{x}, \partial_{t}\right)$ be given by

$$
\widetilde{L}\left(x, \iota ; \partial_{x}, \partial_{t}\right)=J^{-1}\left(\partial_{x}\right) L\left(x, t ; \partial_{x}, \partial_{t}\right) J\left(\partial_{x}\right) .
$$

Then it is easy to see that $L$ is equivalent to $\widetilde{L}$.

Now let us give our results in this note.

Theorem 2. Let the system $L\left(x, t ; \partial_{x}, \partial_{t}\right)$ given by $(1.1)$ be Kowalevskian in Volevič's sense. Then L can be reduced to the first order system by repeated use of the equivalent transformation defined above.

Moreover from the proof of the theorem we can see that the characteristic polynomial of $L$ is preserved under such transformations in a sense. In fact, let $L$ be equivalent to the following first order system,

$$
M\left(x, t ; \partial_{x}, \partial_{t}\right)=\partial_{t} I_{N+l}-\sum_{j=1}^{n} A_{j}(x, t) \partial_{x_{j}}+B(x, t),
$$

where $A$, and $B$ are $(N+l) \times(N+l)$ matrices. Then we have

Theorem 3. Under the assumption in Theorem 2, we have

$$
\operatorname{det}\left(\tau I_{N+l}-\sum_{j=1}^{n} A_{j} \xi_{j}\right)=\tau^{l} p(x, t ; \xi, \tau),
$$

where $p(x, t ; \xi, \tau)$ is the characteristic polynomial of $L$ in Volevičs's sense.

Remember the definition of characteristic polynomial $p$ of $L$ in Volevič's sense, Let $\stackrel{p}{i}_{i j}\left(x, t ; \partial_{x}\right)$ be the homogeneous part of order $t_{i}-t_{j}+1$ of $p_{i j}$. Then the characteristic polynomial $p$ of $L$ is defined by

$$
p(x, t ; \xi, \tau)=\operatorname{det}\left(\tau I_{N}-\left(\AA_{i j}(x, t ; \xi)\right)\right) .
$$

At the end, we note that the reduction of the (single) Kowalevskian equation to the first order system preserving the characteristic polynomial in our sense was considered also by S. Alinhac [3]. 


\section{§ 2. Reduction of the Problem to the Calleulus of Matrix}

As will be shown in this section, our problem is reduced to the calculus of matrix with components of integers. In order to introduce the calculus of matrix, we give two elementary transformations of systems.

(I) Let

$$
p_{i j}\left(x, t ; \partial_{x}\right)=a(x, t) \partial_{x}^{\alpha}+q_{i j}\left(x, t ; \partial_{x}\right), \quad i \neq j .
$$

We put $\alpha=\alpha^{\prime}+\alpha^{\prime \prime}$,

$$
\widetilde{L}\left(x, t ; \partial_{x}, \partial_{t}\right)=\partial_{t} I_{N+1}-\left(\begin{array}{ccccc}
p_{11} & p_{12} \cdots & p_{1 N} & 0 \\
\cdots \cdots \cdots \cdots \cdots \cdots & 0 \\
p_{i 1} & p_{i 2} \cdots & p_{i N} & a \partial_{x}^{\alpha^{\prime}} \\
\cdots \cdots \cdots \cdots \cdots \cdots & 0 \\
p_{N 1} & p_{N 2} \cdots & p_{N N} & 0 \\
0 & 0 & \cdots \cdots & 0 & 0
\end{array}\right)
$$

and

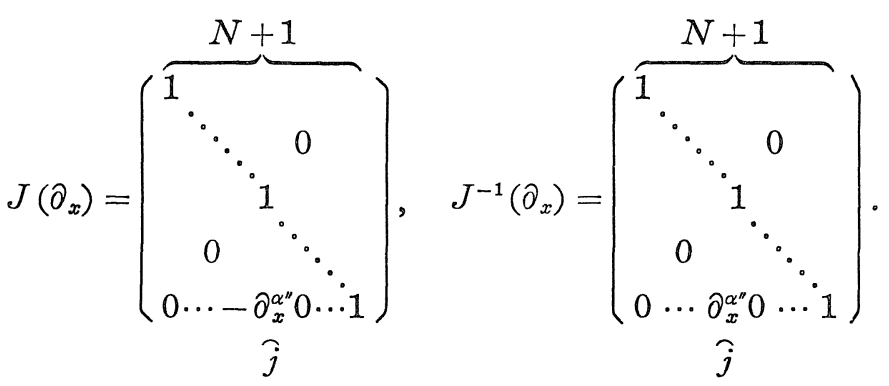

then we obtain

$$
J^{-1} \widetilde{L} J=\partial_{t} I_{N+1}-\left(\begin{array}{ll}
p_{11} \cdots \cdots \cdots p_{1 N} & 0 \\
\cdots \cdots \cdots \cdots \cdots \cdots \cdots & 0 \\
p_{i 1} \cdots q_{i j} \cdots p_{i N} & a \partial_{x}^{\alpha^{\prime}} \\
\cdots \cdots \cdots \cdots \cdots \cdots & 0 \\
p_{N 1} \cdots \cdots \cdots p_{N N} & 0 \\
\partial_{x}^{\alpha^{\prime \prime}} p_{j 1} \cdots \partial_{x}^{\alpha^{n}} p_{j N} & 0
\end{array}\right)
$$

We note that $J^{-1} \widetilde{L} J$ is Kowalevskian in Volevič's sense if $L$ is so. In fact it suffices to choose $t_{N+1}$ so that $t_{j}+\left|\alpha^{\prime \prime}\right| \leqq t_{N+1} \leqq t_{i}+1-\left|\alpha^{\prime}\right|$, 
where $|\alpha|=\left|\alpha^{\prime}\right|+\left|\alpha^{\prime \prime}\right| \leqq t_{i}-t_{j}+1$. Let $p(x, t ; \xi, \tau)$ and $\widetilde{p}(x, t ; \xi, \tau)$ be characteristic polynomials of $L$ and $J^{-1} \widetilde{L} J$ respectively. Then it is clear that $\tilde{p}=\tau p$. Let us now remark that the principal part of the system in Volevič's sense depends on the choice of admissible system $\left\{t_{i}\right\}$ of integers, but its characteristic polynomial does not depend on the choice of them.

Now let us return to our problem. It is obvious that by continuing the above transformations, we can lower the order of $(i, j)$ component. In fact, there exists $m \in\{1,2, \cdots\}$ such that $L$ is equivalent to the following system,

$$
\partial_{t} I_{N+m}-R\left(x, t ; \partial_{x}\right), \quad R=\left(r_{i j}\left(x, t ; \partial_{x}\right)\right)_{i, j=1, \cdots, N+m},
$$

where

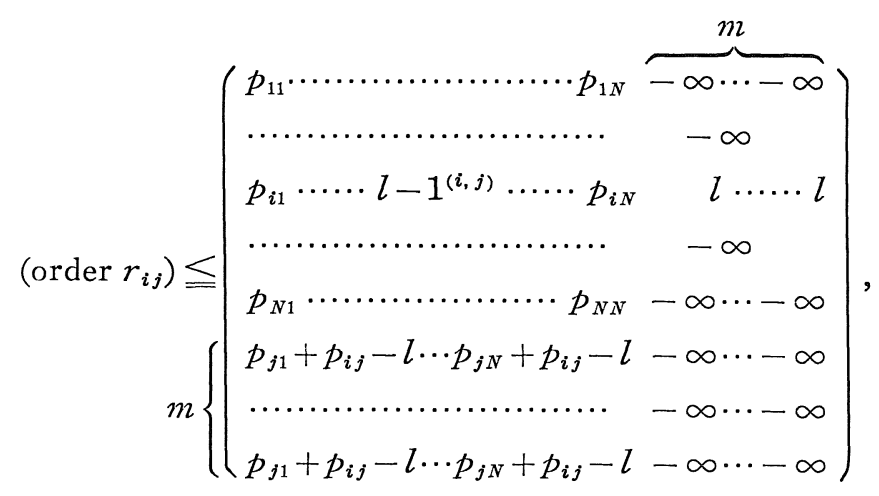

where $p_{i j}=\operatorname{order} p_{i j},-\infty=\operatorname{order}(0), l-1^{(i, j)}=$ the order of $(i, j)$ component is $l-1$, and $\left(r_{i j}\right) \leqq\left(q_{i j}\right),\left(r_{i j}, q_{i j} \in Z\right)$ means $r_{i j} \leqq q_{i j}$ for any $i, j$.

In fact, it suffices to choose $\alpha^{\prime}$ so that $\left|\alpha^{\prime}\right|=l$.

(II) Let

$$
p_{i i}\left(x, t ; \partial_{x}\right)=a(x, t) \partial_{x}^{\alpha}+q_{i i}\left(x, t ; \partial_{x}\right) .
$$

We put $\alpha=\alpha^{\prime}+\alpha^{\prime \prime}$,

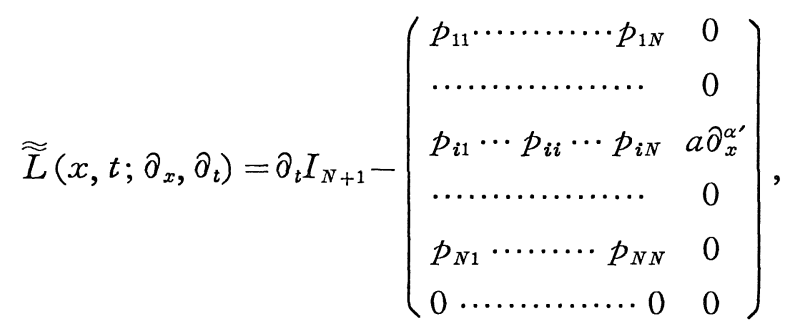


and

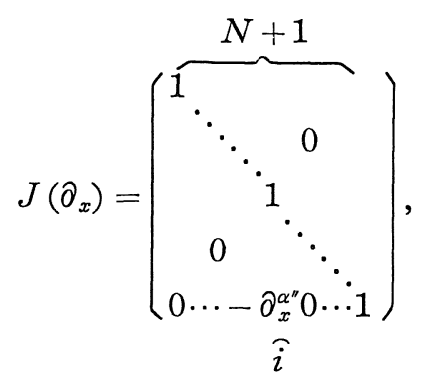

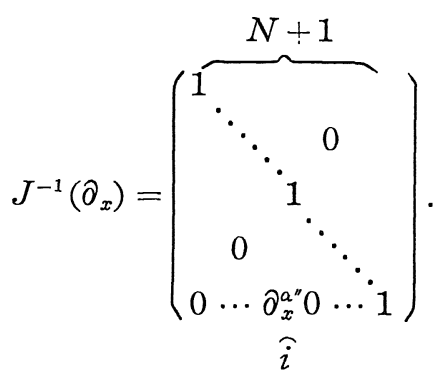

Then we have

$$
J^{-1} \widetilde{\widetilde{L} J}=\partial_{t} I_{N+1}-\left(\begin{array}{ccc}
p_{11} \cdots \cdots \cdots \cdots \cdots & p_{1 N} & 0 \\
\cdots \cdots \cdots \cdots \cdots \cdots \cdots & 0 \\
p_{i 1} \cdots \cdots \cdots q_{i i} \cdots \cdots & p_{i N} & a \partial_{x}^{\alpha^{\prime}} \\
\cdots \cdots \cdots \cdots \cdots \cdots \cdots & 0 \\
p_{N 1} \cdots \cdots \cdots \cdots \cdots & p_{N N} & 0 \\
\partial_{x}^{\alpha^{\prime \prime}} p_{i 1} \cdots \partial_{x}^{\alpha^{\prime \prime}} q_{i i} \cdots & \partial_{x}^{\alpha^{\prime \prime}} a \partial_{x}^{\alpha^{\prime}}
\end{array}\right) .
$$

Therefore, if $L$ is Kowalevskian in Volevič's sense, then $J^{-1} \widetilde{\widetilde{L}} J$ is so. In fact, it suffices to choose $t_{N+1}$ so that $t_{i}+\left|\alpha^{\prime \prime}\right| \leqq t_{N+1} \leqq t_{i}+1-\left|\alpha^{\prime}\right|$, where $|\alpha|=\left|\alpha^{\prime}\right|+\left|\alpha^{\prime \prime}\right| \leqq 1$. And also it is obvious that $\widetilde{p}=\tau p$, where $p$ and $\widetilde{p}$ are the characteristic polynomials of $L$ and $J^{-1} \widetilde{\widetilde{L}} J$ respectively.

Therefore by continuing the above transformations we can lower the order of $(i, i)$ component. In fact, there exists $m \in\{1,2, \cdots\}$ such that $L$ is equivalent to the following system,

$$
\partial_{t} I_{N+m}-S\left(x, t ; \partial_{x}\right), \quad S=\left(s_{i j}\left(x, t ; \partial_{x}\right)\right)_{i, j=1, \cdots, N+m},
$$

where

(2. 12)

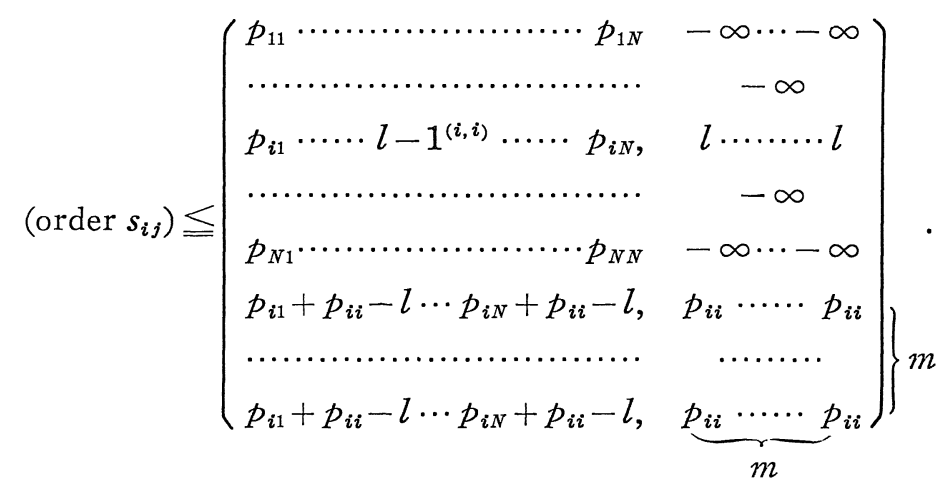


In fact, it suffices to choose $\alpha^{\prime}$ so that $\left|\alpha^{\prime}\right|=l$.

In the following, we shall prove Theorem 2 by the transformations defined above. Hence, by Theorem 2, Theorem 3 is obvious.

Inspired from the above calculus we introduce the following calculus in the matrix with component in $\{0,1,2, \cdots\} \cup\{-\infty\}$.

Let

$$
R=\left(r_{i j}\right), \quad r_{i j} \in\{0,1,2, \cdots\} \cup\{-\infty\} .
$$

$\left(\right.$ I) ${ }^{\prime}$ If $i \neq j$,

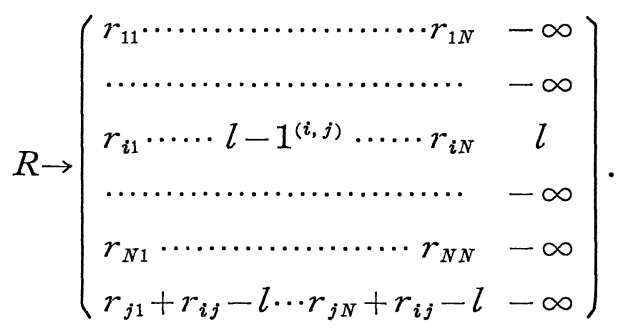

(II)' If $i=j$,

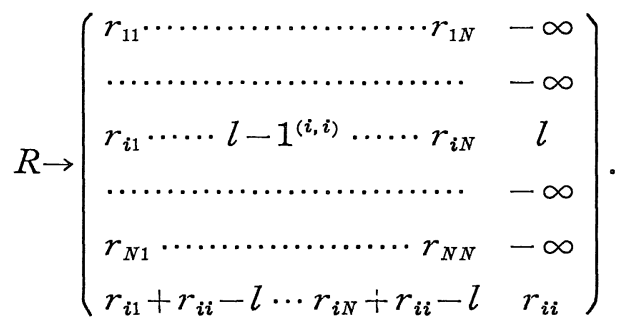

Thus Theorem 2 is reduced to the following,

Theorem 4. Let $R=\left(r_{i j}\right)_{i, j=1, \cdots, N}, \quad r_{i j} \in\{0,1,2, \cdots\} \cup\{-\infty\}$. If $r_{i j} \leqq t_{i}-t_{j}+1$ for any $i, j=1, \cdots, N$ for some $\left\{t_{i}\right\}_{i=1}^{N} \subset \boldsymbol{Z}$, then by the above transformations $(I)^{\prime}$ and $(I I)^{\prime}, R$ can be reduced to a matrix with components $\leqq 1$.

This theorem will be proved in Section 3, but we shall give here some remarks.

In order to prove our theorem we may assume without loss of generality that 


$$
t_{1} \leqq t_{2} \leqq \cdots \leqq t_{N}
$$

by changing rows and columns if necessary. Moreover by joining together many blocks we may assume without loss of generality that $R$ has the following form,

$$
R=\left(\begin{array}{ccccc}
1 & 0 & -\infty & \cdots & -\infty \\
2 & 1 & 0 & -\infty \\
\vdots & \vdots & \ddots & \ddots & -\infty \\
\vdots & \vdots & \ddots & \ddots & \ddots \\
N-1 & \cdots & \cdots \cdots & 0 & 0 \\
N & \cdots & \cdots \cdots & 2 & 1
\end{array}\right)
$$

\section{$\S 3$. Proof of Theorem 4}

As mentioned in the previous section, we consider the matrix $R$ of (2.15) as the original one.

\section{Lemma 3. 1.}

(3. 1)

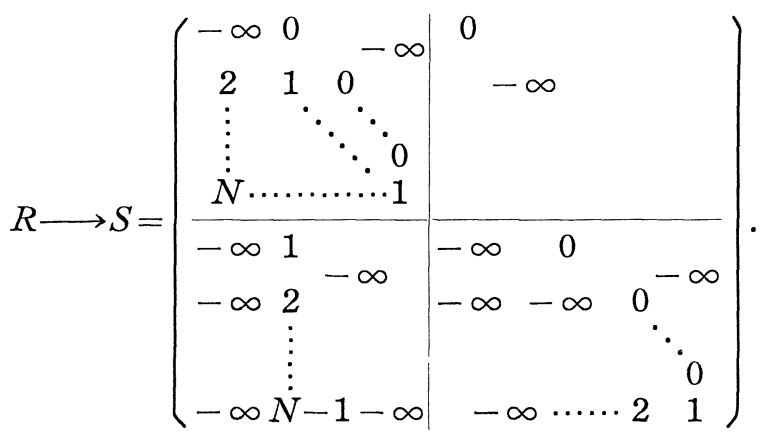

Proof.

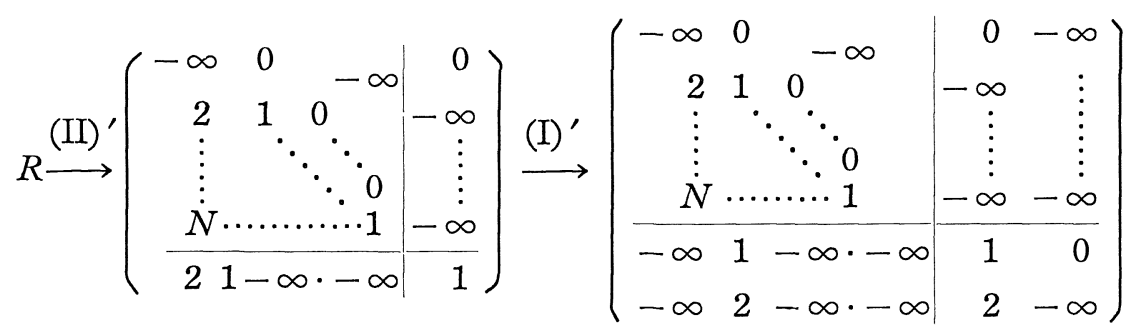




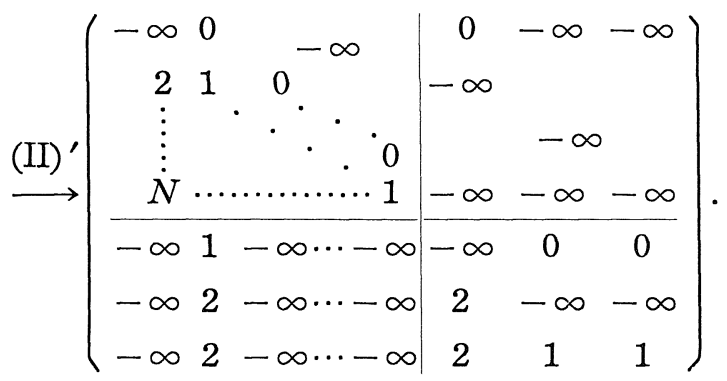

The last matrix can be expressed as follows (joining together the last two rows and columns),

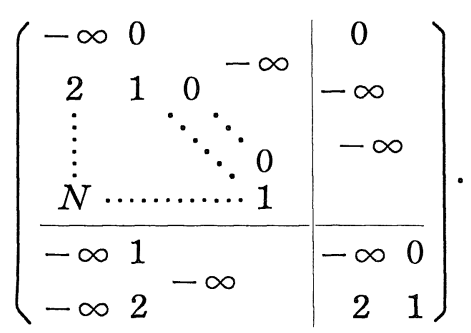

Hence by continuing these operations we have the desired matrix $S$. We note that in the following we use the same simplicity for the brevity.

Q.E.D.

\section{Lemma 3. 2.}

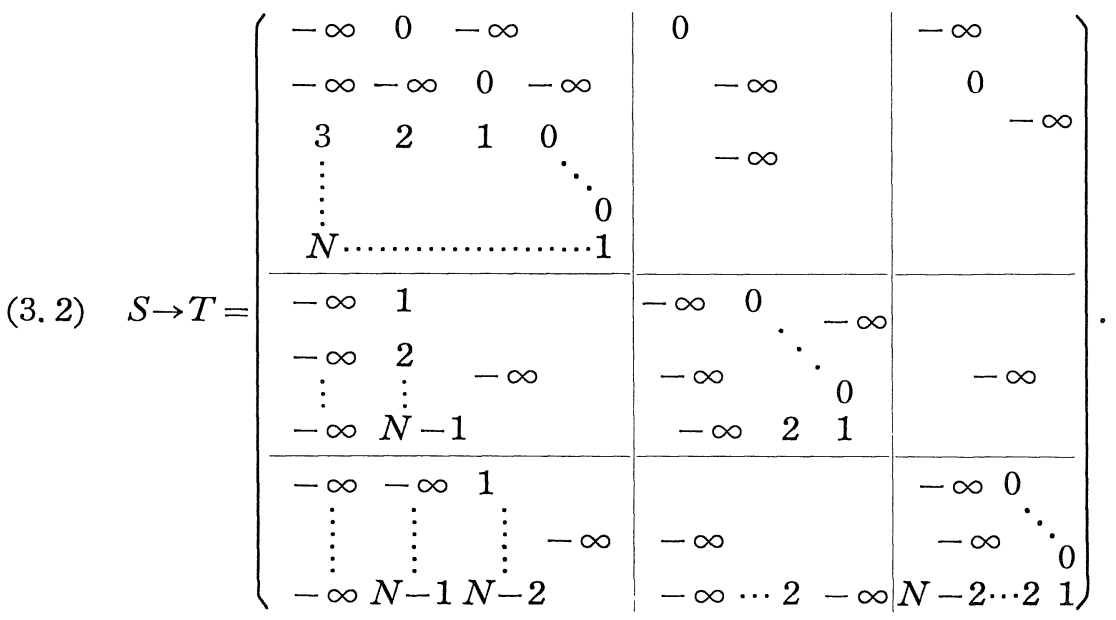

Proof. 


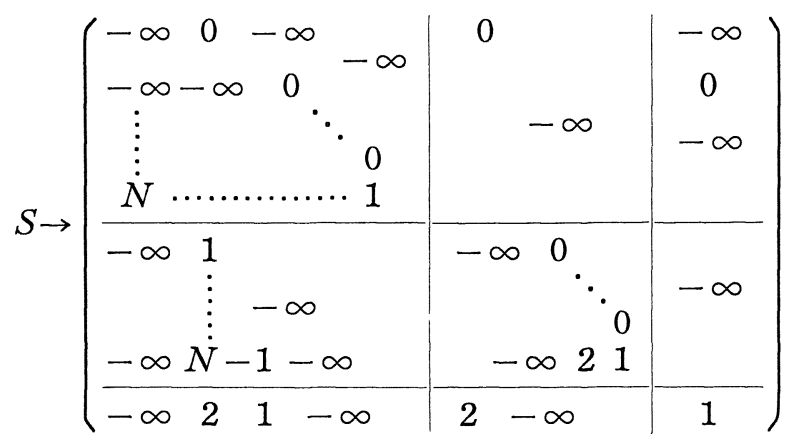

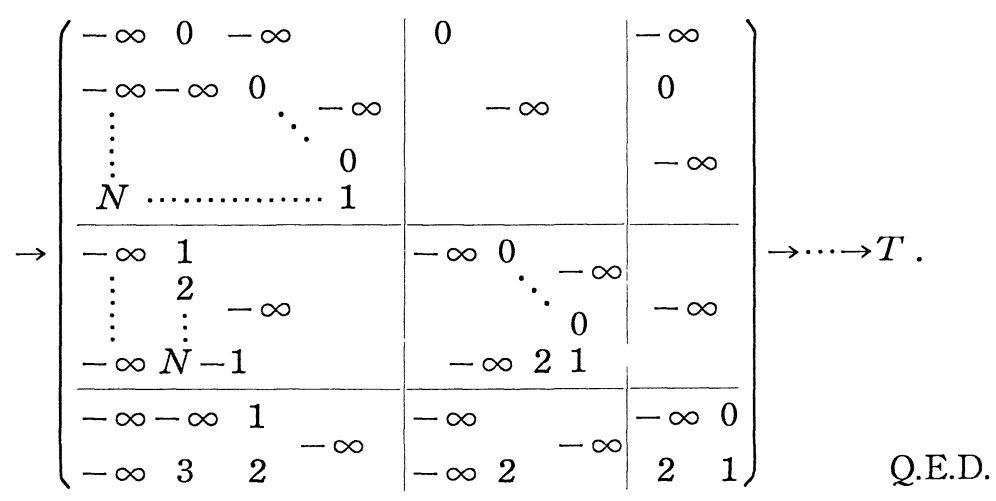

By continuing these transformations, we have

\section{Lemma 3. 3.}

$$
R \rightarrow U=\left(U_{i j}\right)_{i, j=1, \ldots, N},
$$

where $U_{i j}$ are $(N-i+1) \times(N-j+1)$ matrices and

(i)

$$
U_{i i} \leqq\left(\begin{array}{cccc}
-\infty & 0 & & \\
& \ddots & & \\
-\infty & & \ddots & \\
& & -\infty & 0 \\
N-i+1 & \cdots & 2 & 1
\end{array}\right), \quad i=1,2, \cdots, N,
$$

where the equality holds for each $i$ such that $i \neq 2$.

(ii)

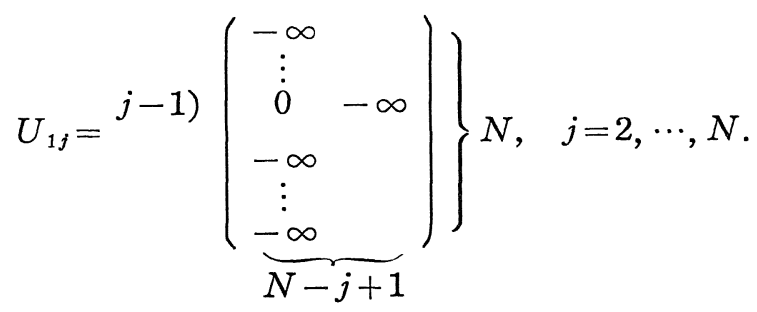




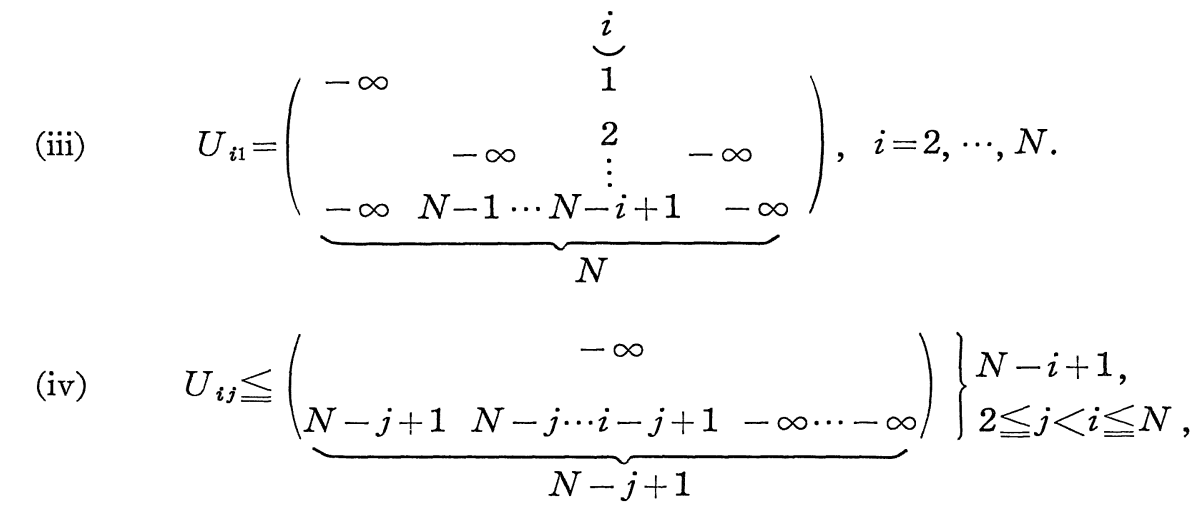

where the equality holds for each $j$ such that $j \neq 2$.

(v)

$$
U_{i j}=(-\infty), \quad 2 \leqq i<j \leqq N
$$

We omit its proof, since it is proved by induction.

Our purpose is to prove that the matrix $U$ in the above lemma can be transformed to the one with components $\leqq 1$. First, we give a simple matrix which is derived from single Kowalevskian equation.

\section{Lemma 3. 4 .}

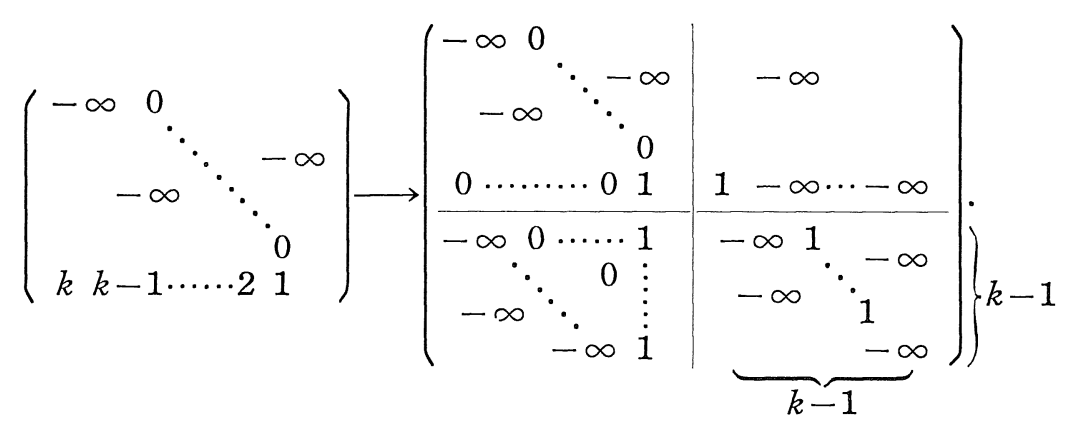

Proof.

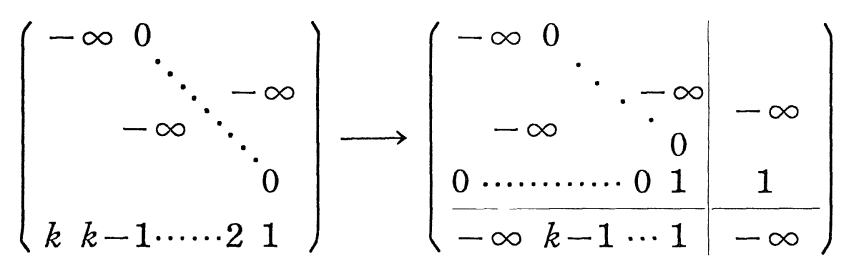




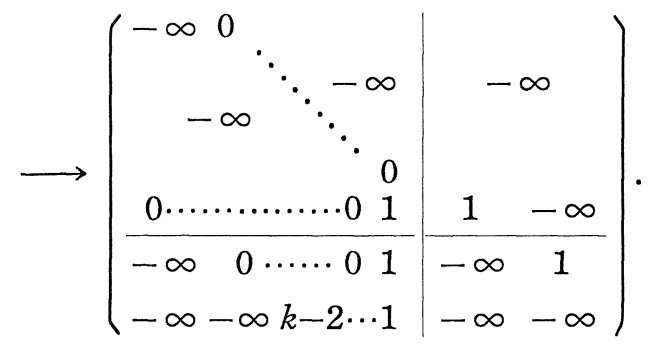

Therefore by continuing these transformations, we obtain our lemma.

Q.E.D.

We remark that the right hand side of (3.4) can be expressed as

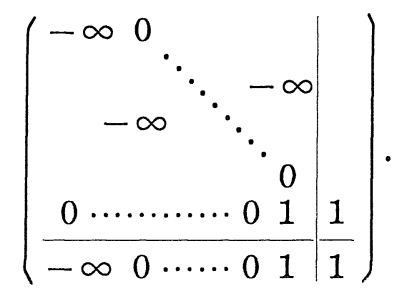

In the following, we shall use the similar abridgments.

Now let $U$ be the one in Lemma 3.3. Then by applying the above lemma we have

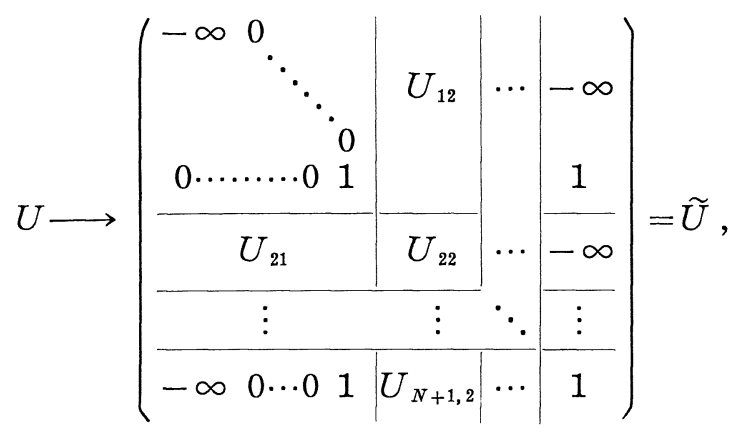

where

$$
U_{N+1, j}=(\underbrace{N-j+1,-\infty, \cdots,-\infty}_{N-j+1}), \quad j=2, \cdots, N
$$

On the other hand, we can lower the numbers in $U_{N+1, j}(j=2, \cdots, N)$ at most 1 . In fact, 


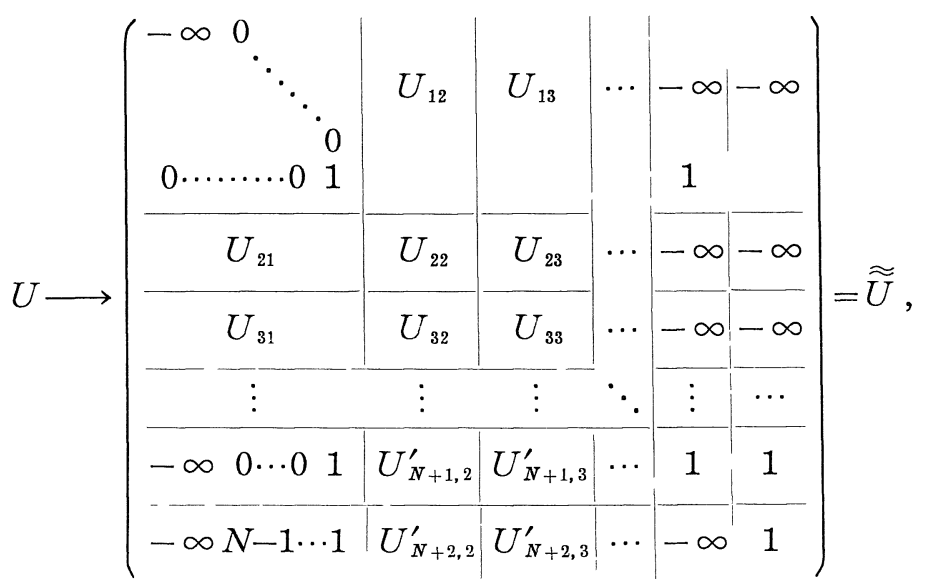

where

$$
\begin{aligned}
& U_{N+1, j}^{\prime}=\overbrace{(0,-\infty, \cdots,-\infty)}^{N-j+1}, \quad j=2, \cdots, N, \\
& U_{N+2, j}^{\prime}=\underbrace{(-\infty, 0, \cdots, 0,1)}_{N-j+1}, \quad j=2, \cdots, N .
\end{aligned}
$$

Thus we have finally a matrix of the following form,

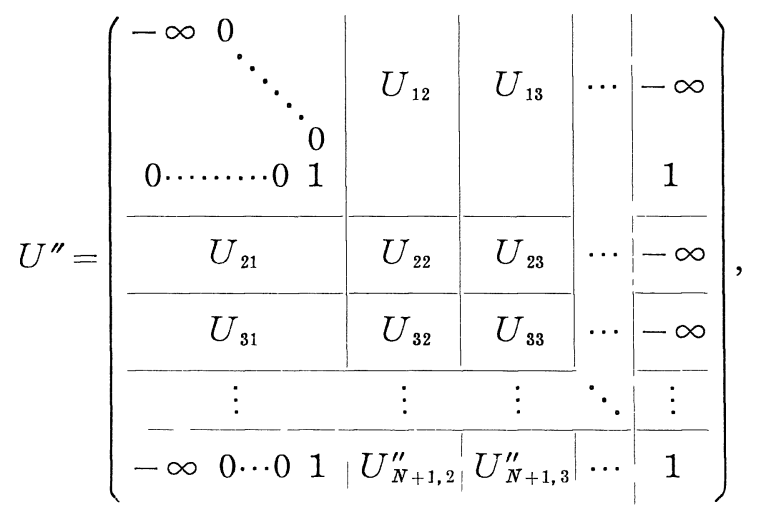

where

$$
U_{N+1, j}^{\prime \prime}=(\underbrace{0, \cdots, 0,1}_{N-j+1}), \quad j=2, \cdots, N .
$$

By continuing these transformations, we have 


$$
U \longrightarrow V=\left(V_{i j}\right)_{i, j=1, \ldots, N+1},
$$

where $V_{i j}$ are $(N-i+1) \times(N-j+1)$ matrices, $(i, j=1, \cdots, N) V_{N+1, j}$ are $1 \times(N-j+1)$ matrices, $(j=1, \cdots, N) \quad V_{i, N+1}$ are $(N-i+1) \times 1$ matrices, $(i=1, \cdots, N)$ and $V_{N+1, N+1}$ is a $1 \times 1$ matrix respectively. Moreover $V_{i j}$ have the following forms,

(i)

$$
\left.V_{i i}=\left(\begin{array}{rrrr}
-\infty & 0 & & \\
& \ddots & \\
& & \ddots & 0 \\
0 \cdots \cdots \cdots & 0 & 1
\end{array}\right)\right\} N-i+1, \quad i=1, \cdots, N, \quad V_{N+1, N+1}=(1)
$$

(ii)

$$
V_{N+1,1}=(\underbrace{-\infty, 0, \cdots, 0,1}_{N}),
$$

(iii)

$$
V_{N+1, j}=(\underbrace{0, \cdots, 0,1}_{N-j+1}), \quad j=2, \cdots, N \text {, }
$$

(iv)

$$
V_{i, N+1}=\left(\begin{array}{c}
-\infty \\
\vdots \\
-\infty \\
1
\end{array}\right), \quad i=1, \cdots, N
$$

(v)

$$
\left.V_{1 j}=j-1\right)\left(\begin{array}{c}
-\infty \\
0 \\
-\infty
\end{array}\right), \quad j=2, \cdots, N
$$

(vi) $\quad V_{i 1}=\left(\begin{array}{ccc}\underbrace{i} & \\ -\infty & 2 & -\infty \\ \vdots & \\ N-i & \\ 0 \cdots & 0 & -\infty \ldots\end{array}\right), \quad i=2, \cdots, N$,

$$
V_{i j} \leqq\left(\begin{array}{c}
-\infty \\
0 \cdots 0
\end{array}\right), \quad 2 \leqq j<i \leqq N
$$

(viii) $\quad V_{i j}=(-\infty), \quad 2 \leqq i<j \leqq N$.

As the final step, under the assumptions on $V$ defined by (3.6), we can lower the numbers in (vi) at most 1 . 
REDUCTION TO THE FIRST ORDER SYSTEMS
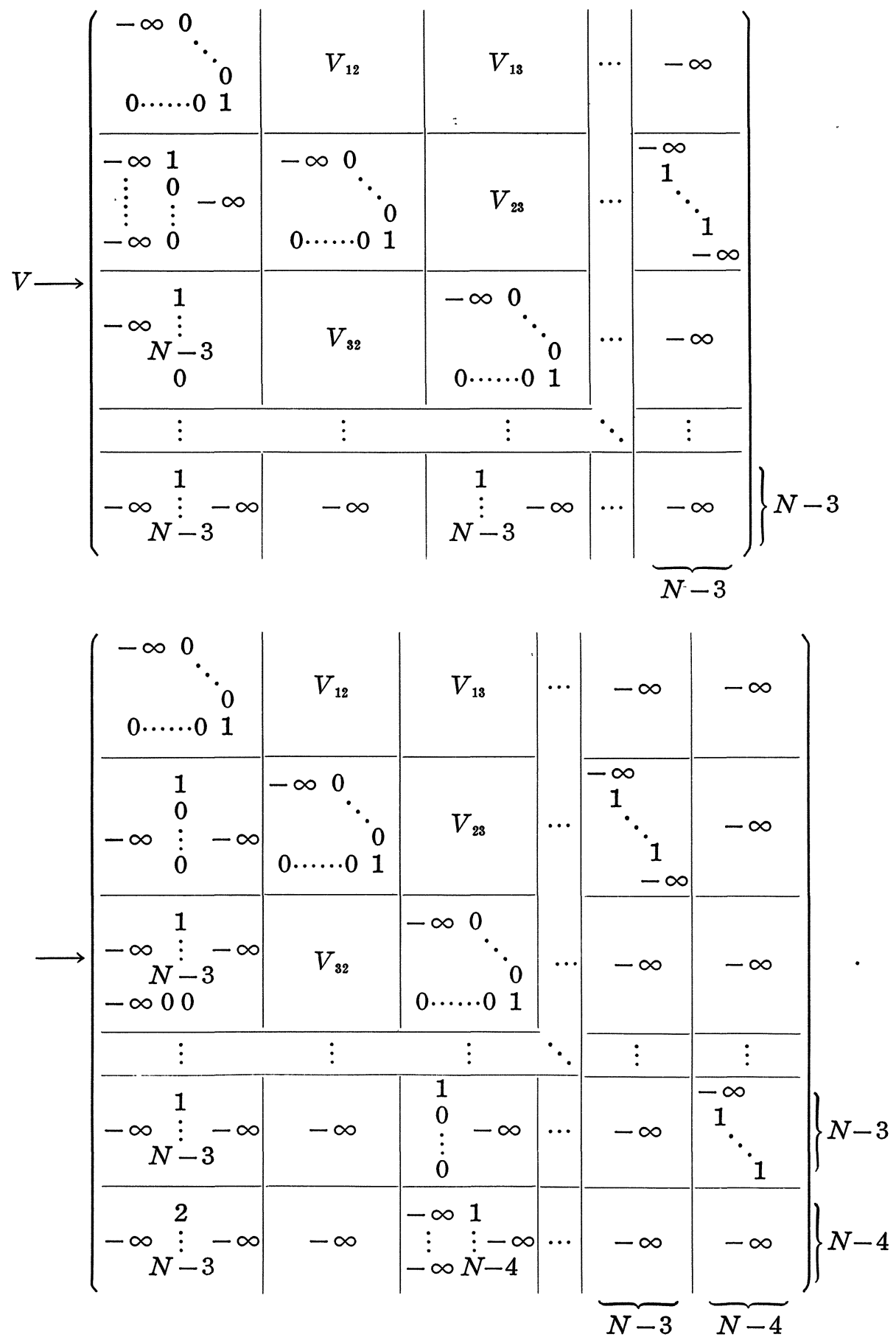

Thus by continuing these operations, Theorem 4 is proved. Q.E.D. 
Example. Let us consider the following system so called CauchyKowalevski's type,

$$
\partial_{t}^{n_{i}} u_{i}=\sum_{j=1}^{N} p_{i j}\left(x, t ; \partial_{x}, \partial_{t}\right) u_{j}, \quad i=1, \cdots, N,
$$

where order $p_{i j} \leqq n_{j}$ and order of $p_{i j}$ with respect to $t<n_{j}$ for any $i, j=1, \cdots, N$.

Now we put

(E. 2) $\quad p_{i j}\left(x, t ; \partial_{x}, \partial_{t}\right)=\sum_{k=1}^{n_{j}} p_{i j}^{(k)}\left(x, t ; \partial_{x}\right) \partial_{t}^{n_{j}-k}, \quad$ order $p_{i j}^{(k)} \leqq k$.

Then if we give $\iota_{i, k}$ by

$$
u_{i, k}=\partial_{t}^{k-1} u_{i}, \quad k=1, \cdots, n_{i}, \quad i=1, \cdots, N,
$$

the system (E. 1) is equivalent to the following one.

(E. 4) $\quad\left\{\begin{array}{l}\partial_{t} u_{i, k}=u_{i, k+1}, \quad k=1, \cdots, n_{i}-1, \quad i=1, \cdots, N, \\ \partial_{t} u_{i, n_{i}}=\sum_{j=1}^{N} \sum_{k=1}^{n_{j}} p_{i j}^{(k)} u_{j, n_{j}-k+1}, \quad i=1, \cdots, N .\end{array}\right.$

If we put the right hand side of (E. 4) by $Q u$, where $u={ }^{t}\left(u_{1,1}, \cdots\right.$, $\left.u_{1, n_{1}}, \cdots, u_{N, 1}, \cdots, u_{N, n_{N}}\right)$, we have

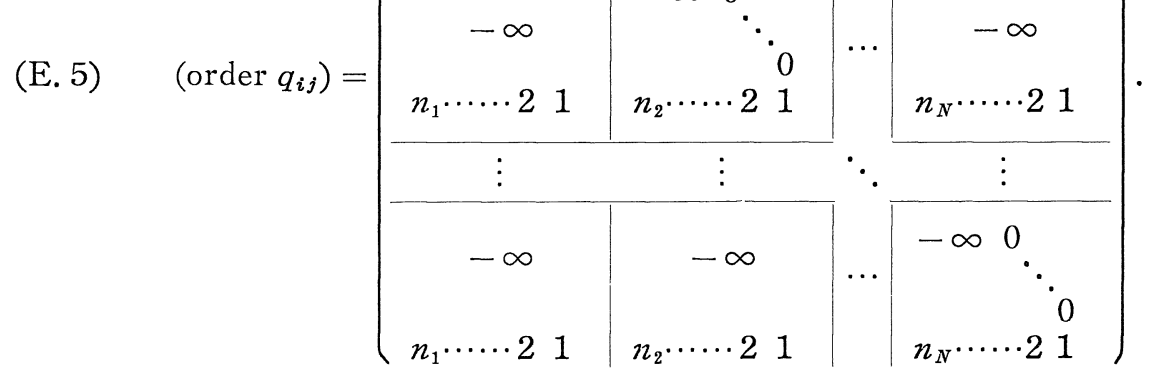

Therefore if we give $\left\{t_{i}\right\}_{i=1}^{n_{1}+\cdots+n_{N}}$ by

(E. 6$) \quad\left\{\begin{array}{l}t_{i}=i, \quad i=1, \cdots, n_{1}, \\ t_{n_{1}+\cdots+n_{k-1}+i}=n_{1}-n_{k}+i, \quad i=1, \cdots, n_{k}, k=2, \cdots, N,\end{array}\right.$ 
it holds obviously that order $q_{i j} \leqq t_{i}-t_{j}+1$ for any $i, j$. In this case, the reduction to the first order system is very easy. In fact,

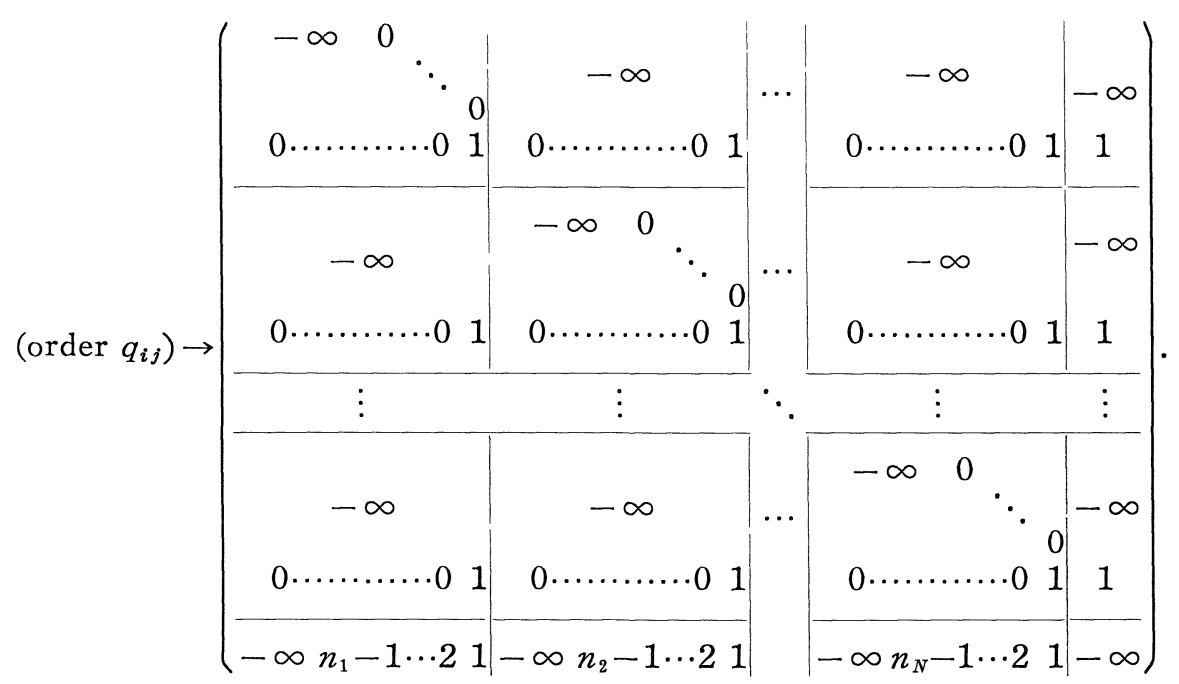

Hence by continuing these transformations, we can reduce to the first order system.

\section{References}

[1] Miyake, M., On Cauchy-Kowalevski's theorem for general systems, Publ. RIMS, Kyoto Univ., 15 (1979), 315-337.

[2] Gårding, L., Une variante de la méthode de majoration de Cauchy, Acta Math., 114 (1965), 143-158.

[3] Alinhac, S., Problèmes de Cauchy pour des opérateurs singuliers, Bull. Soc. Math. France, 102 (1974), 289-315. 
